\title{
Avaliação clínica e radiográfica de alterações ósseas da região distal dos membros torácicos de asininos (Equus asinus) utilizados na tração animal no município de São Luís, MA
}

Clinic and radiographic evaluation of the bone diseases in distal forelimb region of the donkey (Equus asinus) used to traction role in São Luis, MA, Brazil

Evaluación clínica y radiográfica de alteraciones óseas de la región distal de los miembros toráxicos de asininos (Equus asinus) utilizados en la tracción animal en São Luís, MA, Brasil

Flávio Ribeiro Alves ${ }^{1}$; Porfírio Candanedo Guerra² ; Arani Nanci Bomfim Mariana ${ }^{3}$, Luís Carlos Vulcano ${ }^{4}$

Departamento de Cirurgia da Faculdade de Medicina Veterinária e Zootecnia da Universidade de São

Paulo. São Paulo, SP, Brasil 


\section{Resumo}

Objetivo: Realizar um estudo clínico e radiográfico das alterações da estrutura óssea das falanges distais dos membros anteriores direito e esquerdo de asininos utilizados em veículos de tração animal. Material e Método: Foram empregados 17 animais, machos e fêmeas, com idade variando entre 7 e 12 anos, pesando em média $90 \mathrm{Kg}$. Os asininos foram submetidos a exames físico e radiográfico da terceira falange. Resultados: No exame clínico, constataram-se graus de claudicação variando entre 1 e 2, associados a fissuras, e irregularidades no casco; ou ainda alterações no tendão flexor digital superficial e profundo dos dedos, bem como nos ligamentos colaterais, demonstrando o esforço realizado durante as atividades de trabalho. Foram verificadas lesões na sola e ranilha, com comprometimento severo e até destruição em algumas dessas regiões. Radiograficamente, dentre as lesões identificadas, constatou-se osteíte podal em todos os animais, seguida pela destruição e reabsorção óssea da terceira falange $(88,23 \%)$ e pela rotação, fissuras e lises, todas com 23,52\% de ocorrência. Conclusões: Apesar do nível severo das lesões, não houve correlação clínica compatível com os achados observados no exame radiográfico. Portanto, conclui-se que os asininos apresentam grande resistência às atividades físicas para as quais foram selecionados.

Palavras-chaves: Doenças ósseas. Claudicação intermitente. Osteíte. Ossos das extremidades superiores. Falanges. Radiografia. Animais de tração. Equidae.

\section{Introdução e Literatura}

O diagnóstico de claudicações em eqüinos tem representado um desafio para a maioria dos clínicos veterinários, principalmente por advir de um ou mais fatores que em conjunto são observados sob a forma de uma claudicação bem evidente (STASHAK ${ }^{1}, 1994$; DYCE ${ }^{2}$, 1997; POLLITT $^{3}$, 2000).

A claudicação em um dos membros anteriores pode levar a problemas graves no mesmo membro ou no membro anterior contralateral (STASHAK $\left.{ }^{1}, 1994\right)$. Isto se relaciona ao fato de que a massa do eqüídeo localiza-se no meio do seu gradil costal, imediatamente caudal à linha que separa o terço cranial e médio do corpo, deslocando de 60 a $65 \%$ do peso para essa região. Verifica-se ainda, que $95 \%$ das lesões que provocam claudicações estão localizadas na região do carpo ou abaixo dele (COLLES ${ }^{4}$, 1977; COFFMAN ${ }^{5}$, 1983; MOLYNEUX ${ }^{6}$, 1994).

Em estudos radiográficos Thrall ${ }^{7}$ (1998), Butler et al. ${ }^{8}$ (2000) e Becht et al. ${ }^{9}$ ( 2001) observaram osteítes, sesamoidites, rotação de falange, fraturas e calcificação de cartilagens alares como alterações freqüentes da região distal dos membros torácicos de eqüinos, enquanto Kainer ${ }^{10}$ (1989) e Reynolds ${ }^{11}$
(2000) relacionaram tais alterações com a presença de periostites regionais.

Em estudos do osso navicular, Gabriel ${ }^{12}$ (1998) demonstrou a importância do conhecimento da arquitetura de tal osso para a compreensão dos processos patológicos que podem acometer essa região. Em outra pesquisa, Ruohoniemi ${ }^{13}$ (1998) demonstrou um comprometimento da articulação interfalangeana distal com o aparecimento de lesões na borda proximal e nas cartilagens alares da terceira falange, quando foram observadas irregularidades do osso sesamóide distal.

Segundo Melo e Silva ${ }^{14}$ (2002), a ossificação das cartilagens alares é mais freqüente em animais de idade avançada e superior a 12 anos, embora Bengtsson ${ }^{15}$ (1983) tenha relatado sua ocorrência em potros de seis meses de idade. Ruohoniemi ${ }^{13}$ (1998), e Verschooten et al. ${ }^{16}$ (1996) observaram o aparecimento da calcificação da cartilagem alar em cavalos utilizados em trabalhos de tração. Ruohoniemi ${ }^{13}$, em 1998, correlacionou a coexistência freqüiente entre a ossificação da cartilagem alar e processos patológicos do osso navicular.

De acordo com Scott et al. ${ }^{17}$ (1979), Weaver ${ }^{18}$ (1969) e Yovich et al. ${ }^{19}$ (1982), as fraturas de falange distal são consideradas de pouca frequiência. Estudan- 
do 20.638 casos, verificaram que 65 dessas fraturas ocorreram em falange distal e, destas, 57 estavam no membro anterior esquerdo.

Em referências sobre a laminite, Goetz ${ }^{20}$ (1989), Green, et al. ${ }^{21}$ (1991), Hood et al. ${ }^{22}$ (1993), Hunt et al. $^{23}$ (1995) e Tanaka ${ }^{24}$ (2002) descreveram-na como uma doença que promove esquemia das laminas regionais do casco, originando um complexo de doenças causadoras destas alterações. Como resultado dessa complicação, Stick ${ }^{25}$, em 1982, estudando 96 casos de laminite, determinou o prognóstico para esta alteração por intermédio dos g̀raus de rotação da falange distal. Animais que apresentam graus iguais ou menores que $5,5^{\circ}$ estariam aptos a retornarem às atividades esportivas, enquanto aqueles que apresentam rotação superior a $11,5^{\circ}$ estariam impossibilitados.

Na Ilha de São Luís, no Maranhão, os asininos são utilizados em veículos de tração animal, submetendo-se a grandes esforços físicos. Isso resulta muitas vezes em lesões no aparelho locomotor, principalmente em regiões distais dos membros torácicos. Desta forma, a pesquisa teve como objetivo realizar um estudo radiográfico sobre a ocorrência de alterações ósseas que acometem as falanges distais desses animais, bem como relacioná-las à manifestação ou não-manifestação de sinais clínicos.

\section{Material e Método}

Foram utilizados 17 asininos, entre machos e fêmeas, com idade variando de 7 a 12 anos, pesando em média 90 kg, alimentados com ração volumosa, submetidos às mesmas condições de manejo e utilizados em veículos de tração animal. Os animais foram escolhidos aleatoriamente, provenientes de bairros do município de São Luís, MA.

Após a limpeza da região do casco dos membros torácicos direito e esquerdo dos animais, estes foram submetidos ao exame clínico, averiguando-se problemas de deambulação, tais como claudicação a quente, a frio e em seus graus variados. Ao exame físico aferiu-se a temperatura do animal, em seguida, foram realizados exames de palpação para investigar problemas como os de sensibilidade do tendão flexor superfi- cial e profundo dos dedos e ligamentos colaterais. Usando-se uma pinça de casco, aplicou-se pressão sobre a ranilha e a pinça, buscando-se indícios de sensibilidade regional e dor em região do osso navicular.

Em seguida foram realizadas radiografias, baseadas nos protocolos utilizados para equiinos, em projeções dorsoproximal-palmarodistal em ângulos de $45^{\circ} \mathrm{e}$ $65^{\circ}$ e lateromedial, com o animal em estação, utilizando-se um aparelho portátil de Raios-X, MinXrays, modelo HF 100, de 40 - $100 \mathrm{KV}$ p e $20 \mathrm{mAs}$ de potência, calibrado com distância foco-filme de $80 \mathrm{~cm}$ e técnicas de exposição de 45 kVp e 0,5 mAs, chassis metálicos $24 \times 30 \mathrm{~cm}$, com telas intensificadoras CRONEX HI plus e filmes RP-X-OMAT. Os filmes radiográficos foram revelados e fixados manualmente. Assim, os resultados obtidos no estudo radiográfico foram avaliados e correlacionados com os clínicos, sendo a discussão realizada segundo seus achados e os relatos existentes na literatura.

\section{Resultados}

A avaliação clínica revelou a maioria dos animais apresentando claudicação em vários graus, principalmente de grau 1.

O nível de comprometimento do aparelho locomotor dos animais utilizados no estudo variou de moderado a severo, visto que todos apresentavam problemas de aprumo, principalmente devido a um casqueamento inadequado (Figura $1-\mathrm{a}, \mathrm{b}$ e c). Alterações superficiais foram identificadas, tais como sensibilidade dolorosa em talão, ranilha, lesões de sola e edema de coroa. As Tabelas 1 e 2 apresentam uma estimativa percentual dessas lesões nos membros torácicos direito e esquerdo, onde aquelas variam entre 29,41 e $82,35 \%$. Associado a este fato, grande parte dos animais apresentava também algum tipo de alteração que comprometia ligamentos e tendões, verificados, em sua maioria, pela sensibilidade dolorosa nos tendões flexor superficial e profundo dos dedos, bem como nos ligamentos colaterais, demonstrando, dessa forma, os resultados do esforço realizado durante suas atividades de trabalho. Foram verificadas ainda lesões na sola, em alguns casos, inclusive, com 

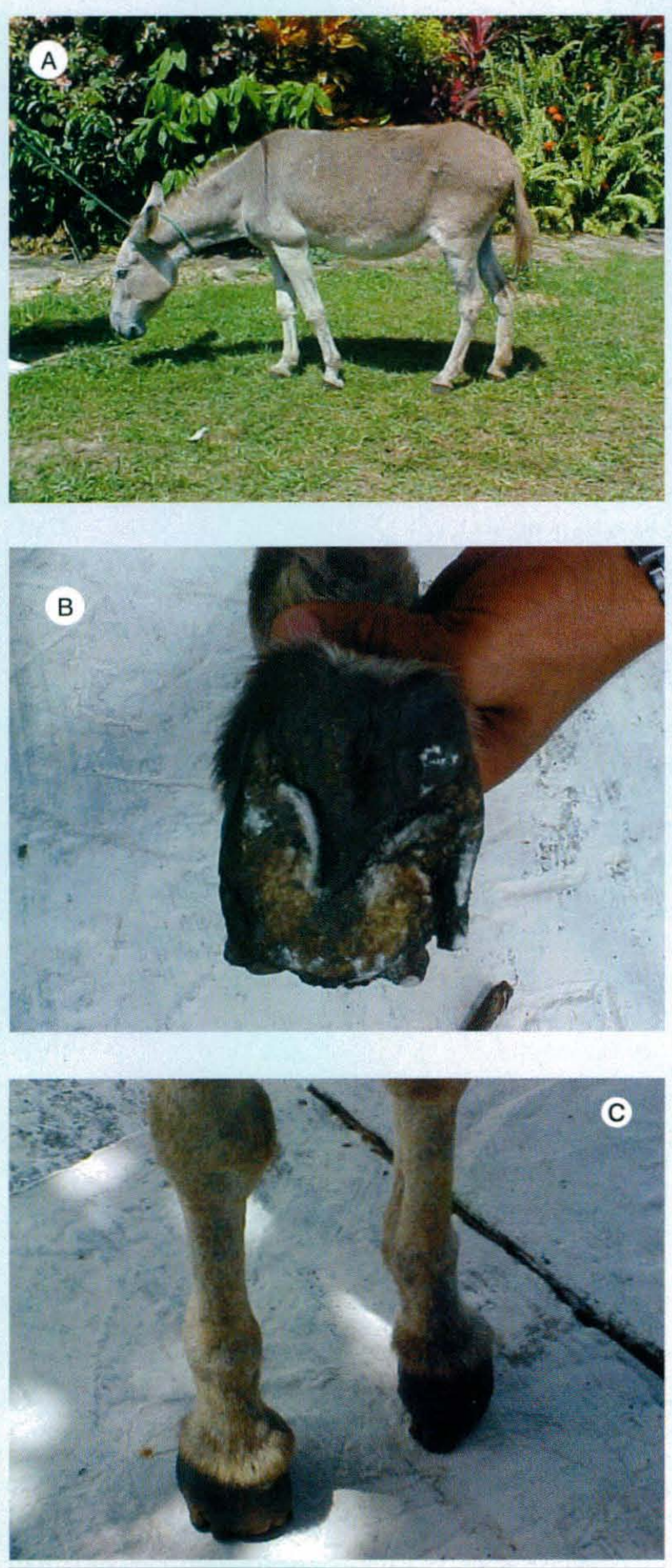

Figura 1 - Irregularidades de casco verificadas como conseqüência de um casqueamento incorreto: (a) dificuldade de aprumo; (b) lesões de sola e destruição da parede do casco; (c) casco de conformação irregular e edema da articulação carpo-metacarpiana
Tabela 1 - Percentual de achados clínicos observados no membro anterior direito de asininos em São Luís, MA, 2003

\begin{tabular}{|lcl|}
\hline Achados clínicos & $\begin{array}{c}\text { No de } \\
\text { lesões }\end{array}$ & $\%$ \\
\hline Fendas no casco & 07 & 41,17 \\
\hline Edema de coroa & 07 & 41,17 \\
\hline Sensibilidade do talão & 14 & 82,35 \\
\hline Sensibilidade da ranilha & 13 & 76,47 \\
\hline Sensibilidade da pinça & 10 & 58,82 \\
\hline Irregularidade do casco & 12 & 70,58 \\
\hline Lesão da sola & 08 & 47,05 \\
\hline Destruição da ranilha & 05 & 29,41 \\
\hline
\end{tabular}

Tabela 2 - Percentual de achados clínicos observados no membro anterior esquerdo de asininos em São Luís, MA, 2003

\begin{tabular}{|lcc|}
\hline Achados clínicos & $\begin{array}{c}\text { No de } \\
\text { lesões }\end{array}$ & $\%$ \\
\hline Fendas no casco & 08 & 47,05 \\
\hline Edema de coroa & 09 & 52,94 \\
\hline Sensibilidade do talão & 14 & 82,35 \\
\hline Sensibilidade da ranilha & 12 & 70,58 \\
\hline Sensibilidade da pinça & 10 & 58,82 \\
\hline Irregularidade do casco & 14 & 82,35 \\
\hline Lesão da sola & 09 & 52,94 \\
\hline Destruição da ranilha & 06 & 35,29 \\
\hline
\end{tabular}

bastante comprometimento desta última. Na ranilha observaram-se alterações que comprometiam grande parte da região. Todos esses achados estão de acordo com informações obtidas durante a anamnese, justificados pelo tipo de esforço que esses animais desenvolvem, já citado anteriormente.

Para a avaliação radiográfica, procurou-se estabelecer uma associação dos resultados dos exames clínicos com os achados radiográficos, já que na rotina hospitalar observam-se animais que apresentam alterações ósseas crônicas, embora na avaliação clínica não 
Tabela 3 - Percentual de achados radiográficos observados no membro anterior direito, por animal, em São Luís, MA, 2003

\begin{tabular}{lcr}
\hline Lesões radiográficas & $\begin{array}{c}\text { No de } \\
\text { les ões }\end{array}$ & \% \\
\hline Osteíte podal & 17 & 100 \\
\hline Fissuras de $3^{\text {a }}$ falange & 04 & 23,52 \\
\hline Rotação de falange & 04 & 23,52 \\
\hline Lises ósseas & 04 & 23,52 \\
\hline Irregularidade em navicular & 05 & 29,41 \\
\hline Destruição e reabsorção da $3^{\text {a falange }}$ & 15 & 88,23 \\
Reação periosteal & 05 & 29,41 \\
\hline Áreas radioluscentes circulares (cistos) & 01 & 5,88 \\
\hline Aumento de espaço articular & 02 & 11,76 \\
\hline
\end{tabular}

Tabela 4 - Freqüência das lesões observadas radiograficamente no membro anterior direito de asininos em São Luís, MA, 2003

\begin{tabular}{lc}
\hline Lesões radiográficas & $\%$ \\
\hline Osteíte podal & 29,31 \\
\hline Fissuras de $3^{\text {a }}$ falange & 6,89 \\
\hline Rotação de falange & 6,89 \\
Lises ósseas & 6,89 \\
\hline Irregularidade em navicular & 8,62 \\
\hline Destruição e reabsorção da $3^{3}$ falange & 25,86 \\
Reação periosteal & 8,62 \\
\hline Áreas radioluscentes circulares (cistos) & 1,72 \\
\hline Aumento de espaço articular & 3,44 \\
\hline
\end{tabular}

se perceba o problema ou este seja verificado com pouca intensidade. A análise das radiografias dos animais utilizados no estudo demonstrou a ocorrência de grande número de alterações podais que acometem os asininos nos membros torácicos. Os valores percentuais para cada membro, demonstrados por animal e por frequiência de aparecimento de cada lesão, foram listados nas Tabelas de 3 a 6.
Tabela 5 - Percentual de achados radiográficos observados no membro anterior esquerdo por animal em São Luís, MA, 2003

\begin{tabular}{lcc}
\hline Lesões radiográficas & $\begin{array}{c}\text { No de } \\
\text { lesões }\end{array}$ & $\%$ \\
\hline Osteíte podal & 17 & 100 \\
\hline Fissuras de $3^{\text {a falange }}$ & 05 & 29,41 \\
\hline Rotação de falange & 05 & 29,41 \\
Lises ósseas & 02 & 11,76 \\
\hline Irregularidade em navicular & 04 & 23,52 \\
\hline Destruição e reabsorção da 3a falange & 09 & 52,94 \\
Reação periosteal & 03 & 17,64 \\
Radioluscência circulares (cistos) & 01 & 5,88 \\
\hline Aumento de espaço articular & 01 & 5,88 \\
\hline
\end{tabular}

Tabela 6 - Freqüência das lesões observadas radiograficamente no membro anterior esquerdo de asininos em São Luís, MA, 2003

\begin{tabular}{|lc}
\hline Lesões radiográficas & $\%$ \\
\hline Osteíte podal & 36,17 \\
\hline Fissuras de $3^{\text {a }}$ falange & 10,63 \\
\hline Rotação de falange & 10,63 \\
\hline Lises ósseas & 4,25 \\
\hline Irregularidade em navicular & 8,51 \\
\hline Destruição e reabsorção da $3^{\text {a falange }}$ & 19,14 \\
\hline Reação periosteal & 6,38 \\
\hline Áreas radioluscentes circulares (cistos) & 2,12 \\
\hline Aumento de espaço articular & 2,1 \\
\hline
\end{tabular}

Todos os animais apresentaram osteíte podal severa, graus variados de reabsorção óssea, havendo alguns casos de lise óssea intensa, principalmente no membro anterior direito (Figura $2-\mathrm{a}$, b e c). A presença de fraturas incompletas da falange, como consequiência de uma sobrecarga das estruturas regionais de sustentação ou traumas, foram observadas com freqüência maior no membro anterior esquerdo, onde três dos sete ani- 

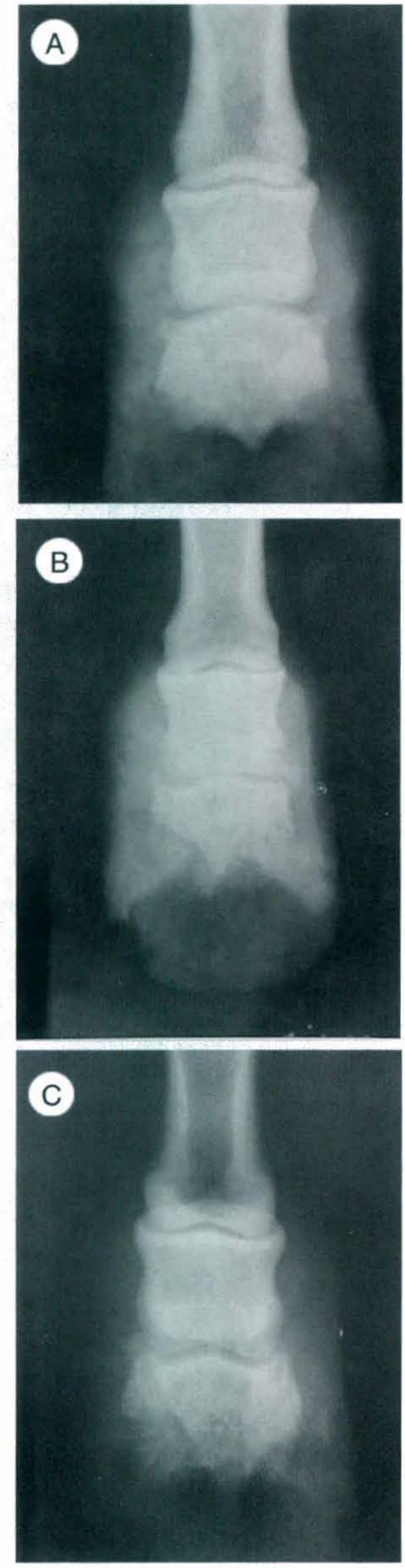

Figura 2 - Imagens radiográficas do membro anterior direito de três animais apresentando osteíte podal severa, com intensa destruição e reabsorção óssea da borda: (a) radiografia dorsopalmar oblíqua com ângulo de $65^{\circ}$ (DPr65PaDiO); (b) e (c) radiografia dorsopalmar oblíqua com ângulo de $45^{\circ}(\mathrm{DPr} 45 \mathrm{PaDiO})$
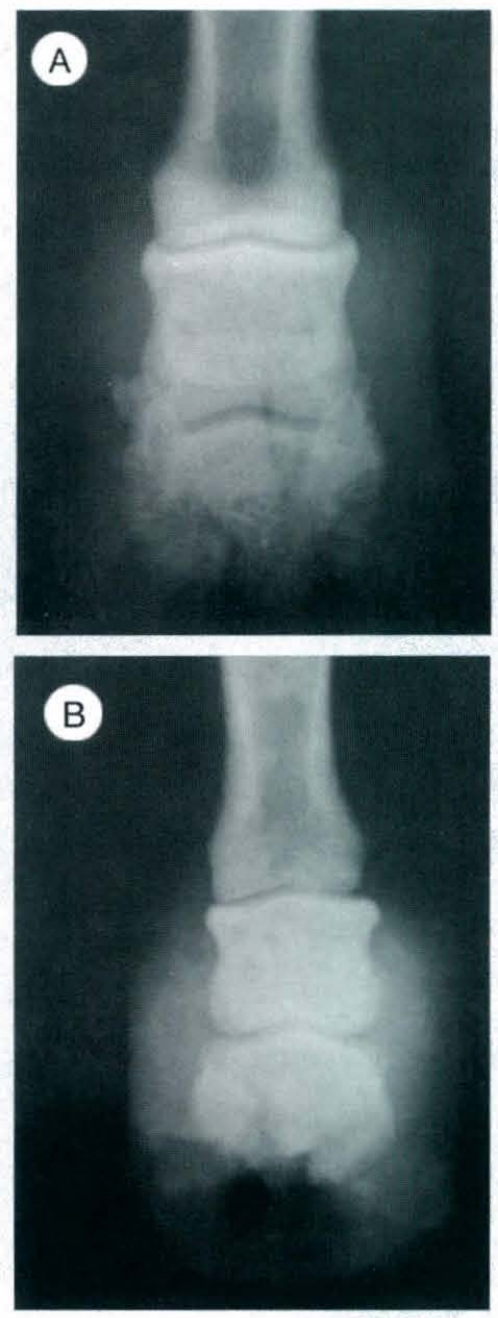

Figura 3 - Imagens radiográficas do membro anterior esquerdo de dois animais que apresentaram problemas de fissuras na falange distal: (a) radiografia dorsopalmar oblíqua com ângulo de $45^{\circ}$; (b) radiografia dorsopalmar oblíqua com ângulo de $65^{\circ}$

mais que apresentaram fissuras também possuíam fendas no casco (Figura 3-a e b). As rotações de terceira falange foram pouco observadas no membro anterior direito, mas em cinco $(29,41 \%)$, dos dezessete animais, verificou-se complicação associada a esta alteração no membro anterior esquerdo, onde os mesmos animais apresentaram intensa sensibilidade dolorosa em região de talão, ranilha e pinça (Figura 4). Casos de lises ósseas foram pouco freqüentes em ambos os membros e entre as amostras, mas quando presentes, associavamse a problemas de destruição e reabsorção óssea, bem como a lesões de sola e destruição da ranilha. 

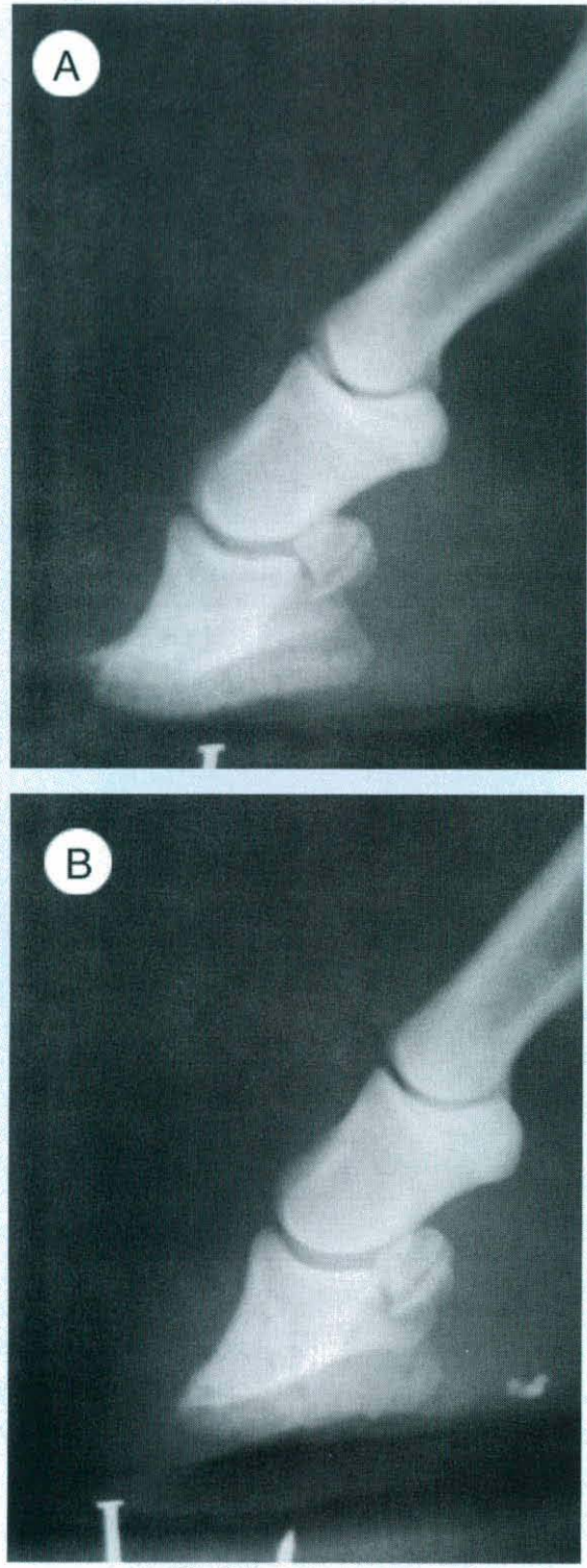

Figura 4 - Radiografia lateral de animais com sinais radiográficos de rotação da $3^{\underline{a}}$ falange

\section{Discussão}

No exame físico realizado nos asininos, verificouse que todos apresentavam certo grau de claudicação, principalmente de grau 1, tanto no membro anterior direito quanto no esquerdo, confirmando as observações feitas por Pollitt ${ }^{26}$ (1992) e Prinz ${ }^{27}$ (1992) em seus estudos sobre as alterações de deambulação na espécie eqüina, atribuídos a possíveis lesões ósseas, de articulações, ligamentos colaterais e às claudicações de apoio, tais como os achados deste trabalho. Da mesma forma, as observações do presente estudo estão de acordo com as de Colles ${ }^{4}$, (1977); Coffman ${ }^{5}$ (1983); Molyneux ${ }^{6}$ (1994), Dyson ${ }^{28}$ (2003), Becht et al..$^{9}$ (2001), quando notificaram que problemas no casco, mesmo sendo de graus variados, provocam alterações na locomoção do animal.

Os achados radiográficos revelaram osteíte podal severa em todos os casos. Contudo, os asininos não apresentaram sintomatologia clinica de acordo com a gravidade da alteração, contrapondo-se aos estudos de Molineux $^{6}$ (1994), Pollitt ${ }^{29}$ (1998), Kainer ${ }^{10}$ (1989); Butler $^{8}$ (2002) e Becht et al. ${ }^{9}$ (2001), que verificaram quadros de osteíte podal com prognóstico desfavorável, associada a reações periosteais na superfície dorsal da terceira falange e remodelamento do processo palmar, situação que impossibilitava os animais acometidos de se locomoverem adequadamente. Essa diferença no comportamento dos animais pode estar relacionada com a espécie ou com o seu peso, já que está de acordo com Gabriel ${ }^{12}$ (1998), quando, estudando a espécie eqüina, verificou que a radiodensidade óssea desses animais era proporcional ao seu peso ou ao peso que carregavam.

Dos dezessete animais utilizados para o experimento, sete apresentaram problemas associados a fissuras na terceira falange $(41,17 \%)$, dois destes bilateralmente. Entretanto, verificou-se uma ocorrência maior da alteração no membro anterior esquerdo em cinco dos sete animais $(29,41 \%)$ que a manifestaram, possivelmente, em decorrência de algum fator de desequilíbrio do aprumo para este lado, conforme estudos de Scott et al. ${ }^{17}$ (1969), Weaver ${ }^{18}$ (1969) e Yovich $^{19}$ (1982). Estes autores, estudando 20.638 casos de eqüinos admitidos em um hospital, observaram que 65 eram de fraturas de falange distal e, destes, 57 correspondiam a fraturas da falange distal esquerda, embora tenham utilizado animais desportistas, uma atividade diferente da executada pelos animais deste estudo. Os mesmos autores consideraram rara a ocor- 
rência desta alteração, no entanto, ela foi observada em $41,17 \%$ dos animais desta pesquisa, assemelhando-se aos achados de Dyson ${ }^{30}$ (1997) e Hood ${ }^{31}$ (1999) quando estudaram a ocorrência de fraturas da falange distal de eqüinos.

A rotação de falange constituiu outra característica, observada em cinco animais $(29,41 \%)$, em um deles, bilateralmente, e associada a aumento do espaço intra-articular. Todos os animais apresentaram rotaçã̉o de falange com angulação variando entre 7 e 10 graus, o que Stick $^{25}$ (1982) notificou como resultados de laminite, impossibilitando os animais para o trabalho. Essa informação vai de encontro às observações da presente pesquisa, posto que, todos os animais utilizados no estudo, inclusive os que apresentavam rotação de falange, estavam em plena atividade de trabalho e não apresentavam graus de claudicação superior a 2, o que mostra resistência superior dos asininos utilizados em veículos de tração. Em todos os animais que se verificou rotação de falange, esta apresentou-se como desvio ventral, fato também observado por Kuwano et al. ${ }^{32}$ (1997), Pollit $^{29}$ (1998), Butler et al. ${ }^{8}$ (2002) e Tanaka et al. ${ }^{24}$ (2002), que em suas pesquisas verificaram uma ocorrência mais alta do deslocamento ventral da falange, quando comparados com o deslocamento paralelo em relação à muralha do casco.

Com o estudo radiográfico, obtendo-se imagens da borda proximal e distal do osso navicular em sete animais avaliados $(41,17 \%)$, foi possível identificar algum tipo de irregularidade, dois deles, inclusive, manifestando a alteração bilateralmente associada a reações periosteais na superfície dorsal do terço proximal da falange distal e processo palmar lateral, coincidindo com os achados de Numans $^{33}$ (1973); Adams ${ }^{34}$ (1974); Lowe ${ }^{35}$ (1976); Ackerman et al. ${ }^{36}$ (1977); Vadez ${ }^{37}$ (1978); Gabriel ${ }^{12}$ (1998) e Ruohoniemi ${ }^{13}$ (1998). Estes autores relataram a presença de processos patológicos da articulação interfalangeana distal e reações periosteais periarticulares em animais com irregularidades do osso navicular, levando, de alguma forma, a algum grau de comprometimento para a falange distal e, conseqüentemente, para o aparelho locomotor destes animais.

Os processos alares dos asininos apresentaramse pouco desenvolvidos, favorecendo a não-ocorrên- cia de calcificação das cartilagens alares nesses animais, informação essa que se contrapõe aos achados de Ruohoniemi ${ }^{13}$ (1998); Verschooten ${ }^{16}$ (1996); Stashak ${ }^{1}$ (1998) e Melo e Silva ${ }^{14}$ (2002) que, estudando a arquitetura do osso navicular em cavalos, observaram calcificações da cartilagem alar em animais de idade avançada, ou submetidos a atividades de tração e transporte.

Os resultados obtidos pelos pesquisadores Kuwano $^{32}$ (1997); Butler et al. ${ }^{8}$ (2000) e Tanaka et al. ${ }^{24}$ (2002) em estudos realizados na espécie eqüina permitiram estimar que a espécie asinina apresenta uma resistência maior aos distúrbios do aparelho locomotor, em especial da falange distal, quando ambos submetem-se às mesmas condições de trabalho.

\section{Conclusões}

Nas condições em que foi realizado o estudo, e de acordo com os métodos de avaliação que foram utilizados, pode-se concluir que:

- Em asininos, a osteíte podal não mostrou correlação clínica com os achados evidenciados na imagem radiográfica;

- As fissuras da falange distal ocorrem mais no membro anterior esquerdo, estando associadas à presença de fendas nos cascos desses animais;

- A rotação da falange distal, mesmo em grau avançado, não mostra sintomas clínicos severos, demonstrando que os asininos apresentam limiar de dor maior quando comparados com os dados bibliográficos de equiinos, ou melhor acomodação do membro ao apoiar.

Os achados radiográficos de irregularidades do bordo dorsal do osso navicular e as reações periosteais não necessariamente demonstraram sintomatologia clínica evidente durante o exame físico, com os asininos apresentando maior resistência quando estas mesmas alterações são observadas em eqüinos, segundo os dados bibliográficos consultados.

Em asininos, as cartilagens colaterais apresentaram-se, radiograficamente, muito rudimentares, mesmo nos animais mais idosos e submetidos a atividades físicas intensas. 


\section{Abstract}

Objective: To perform a clinical and radiographic study of the bone alterations of the distal phalanx, of the right and left anterior limbs of asinine used in animal traction vehicles. Material and Method: Survey included 17 animals, male and female, ages ranging from seven to twelve years, weighing in average $90 \mathrm{~kg}$. Asinines were submitted to physical and radiological tests of the third phalanx. Results: The clinical examination detected levels of lameness varying from 1 to 2, associated to fissures and hooves abnormality, and also alterations of the deep and superficial digital flexor tendon of the digits, as well as the collateral ligament, demonstrating the effect during work activities. Lesions of the sole and frog, in some cases severe and even with destruction of said regions. According to radiographic data the following lesions were identified: podal osteitis (100\%); bone destruction and reabsorption of the third phalanx (88,23\%) each. Conclusions: Despite of the severity of the lesions, there was no correlation between the clinical and radiographic findings. We concluded that the asinines are more resistant than the equines when submitted to the same physical activities.

Keywords: Bone diseases. Laminitis. Osteitis. Limb bones. Phalanges. Radiography. Working animals. Donkeys.

\section{Resumen}

Objetivo: Realizar un estudio clínico y radiográfico de las alteraciones en la estructura ósea de las falanges distales, de los miembros anteriores derecho e izquierdo, de asininos utilizados en vehículos de tracción animal. Material y Método: Fueron utilizados 17 animales, machos y hembras, teniendo entre siete y doce años y pesando como promedio $90 \mathrm{Kg}$. Los asininos pasaron por exámenes físicos y radiográficos de la falange tercera. Resultados: En el examen clínico se constataron grados de claudicación, oscilando entre 1 y 2, asociados a fisuras, e irregularidades en el casco o aún, alteraciones en el tendón flexor digital superficial y profundo de los dedos, así como en los ligamentos colaterales, demostrando el esfuerzo realizado durante las actividades de trabajo. Se verificaron lesiones en la planta y "rana", con lesiones graves e incluso destrucción en algunas de esas regiones. Radiograficamente, entre las lesiones observadas, se constató osteítis podal en todos (100\%) los animales, acompañada de destrucción y reabsorción ósea de la falange tercera $(88,23 \%)$ y por rotación, fisuras y lisis, todas con $23,52 \%$ de ocurrencia. Conclusiones: A pesar del nivel grave de las lesiones, no hubo correlación clínica compatible con lo que se observó en el examen radiográfico, y la conclusión fue que los asininos presentan gran resistencia para las actividades físicas para las que fueron escogidos.

Palabras-claves: Enfermedades óseas. Claudicación intermitente. Osteítis. Huesos de las extremidades superiores. Falanges. Radiografía. Animales de tracción. Equidae.

\section{Referências}

1. STASHAK, T. Claudicação em eqüinos segundo Adams. 4. ed. São Paulo: Roca, 1994. p. 503-558.

2. DYCE, K. M; SACK, W. O; WENSING, C. J. G. Tratado de anatomia veterinária. Rio de Janeiro: Guanabara Koogan, 1997. p. 171-183.
3. POLLITT, C. C. Clinical anatomy and physiology of the normal equine foot. Equine Veterinary Education, v. 4, p. 219-224, 2000.

4. COLLES, C. M.; LEFFCOTT, L. B. Laminitis in the horse. Veterinary Record, v. 100, p. 262-264, 1977. 
5. COFFMAN, J. R. Acute laminitis: mechanisms and therapy. In: BAIN-FALLON MEMORIAL LECTURES, 6., 1983, Sydney. Sydney: University of Sydney, 1983. Proceedings... Equine intemal medicine, p. 68-72.

6. MOLYNEUX, G. S. et al. The structure, innervation and location of arteriovenous anastomoses in the equine foot. Equine Veterinary Journal, v. 4, p. 305-312, 1994.

7. THRALL, D. E. Textbook of veterinary diagnostic radiology. 3. ed. Philadelphia: Saunders, 1998. p. $242-246$.

8. BUTLER, J. A. et al. Clinical Radiology of the horse. 2. ed. Malden: Blackwell, 2000. p. 27-82.

9. BECHT, James L. et al. Radiographic interpretation of normal skeleton variations and pseudolesions in the equine foot. The Veterinary Clinics of North America: Equine Pratice, v. 17, p. 1-19, 2001.

10. KAINER, R. A. Clinical anatomy of the equine foot. The Veterinary Clinics of North America: Equine Practice, v. 1, p. 1-27, 1989.

11. REYNOLDS, J. A. Nutrition and developmental orthopedic disease in horses. Feed Facts Horse, p. 3 - 4, Jan. 2000.

12. GABRIEL, A. et al. Morphometric study of the equine navicular bone: variations with breeds and types of horse and influence of exercice. Journal of Anatomy, v. 7, p. 535-539, 1998.

13. RUOHONIELMI, M. et al. Radiographic appearance of the navicular bone and distal interphalangeal joint and their relationship with ossification of the collateral cartilages of the distal phalanx in finnerhorse cadaver forefeet. Veterinary Radiology and Ultrasound, v. 39, p. 125-132, 1998.

14. MELO e SILVA, S. R. A.; VUlCANO, L. C. Collateral cartilage ossification of the distal phalanx in the Brazilian jumper horse. Veterinary Radiology and Ultrasound, v. 43, p. 461- 463, 2002.

15. BENGTSSON 1983 apud $^{14}$.
16. VERSCHOOTEN, F. et al. The Ossification of cartilages of distal phalanx in horse. Journal Equine Veterinary Science, v. 7, p. 291-305, 1996.

17. SCOTT, E. A. et al. A review of third phalanx fractures in horse. Journal of the American Veterinary Medical Association, v. 174, p. 1337$1343,1979$.

18. WEAVER, A. D. Fracture of equine pedal bone. Equine Veterinary Journal, v. 1, p. 283-286, 1969.

19. YOVICH, J. V. et al. Fractures of distal phalanx in horse. Australian Veterinary Journal. n.58, p. 180, 1982.

20. GOETZ, T. E. The treatement of laminitis in horses. The Veterinary Clinics North America: Equine Pratice, v. 5, p. 73-108, 1989.

21. GREEN, E. M. et al. Equine medicine and surgery. 4. ed. Goleta: American Veterinary Publications, 1991. v. 2. p. 1354-1366.

22. HOOD, D. M. et al. The role of vascular mechanisms in the development of acute laminitis. Journal of Veterinary Internal Medicine, v. 7, p. 228-234, 1993.

23. HUNT, R. J. et al. Diseases of the foot. Philadephia: Saunders, 1995. p. 659-705.

24. TANAKA, K. et al. A study of laminitis in racehorses: recovery from prolapse of the solar corium. Journal of Equine Science, v. 13, p. 1-7, 2002 .

25. STICK 1982 apud $^{1}$.

26. POLlitT, C. C. The role of arteriovenous anastomoses in the pathophysiology of equine laminitis. In: ANNUAL CONVENTION OF THE AMERICAN ASSOCIATION OF EQUINE PRACTITIONERS, 37., 1992, San Francisco. Proceedings... San Francisco: American Association of Equines Practitioners, 1992. p. 711720 .

27. PRINZ, L., SMITH, M. Laminitis. Equus, v. 13, p. 56-61, 1992. 
28. DYSON, S. et al. Magnetic resonance imaging of the equine foot: 15. Horse. Equine Veterinary Journal, v. 35, p. 18-26, 2003.

29. POLLITT, C. C., DARADKA, M. Equine basement membrane pathology: loss of type IV collagen, type VII collagen and laminin immunostainning: the equine hoof. Equine Veterinary Journal, v. 30, p.139144, 1998. Supplement 26.

30. DYSON, S. J. Clínica de eqüinos: perguntas e respostas ilustradas de medicina veterinária. São Paulo: Manole, 1997. p 38-52.

31. HOOD, D. M. Laminitis in horse. Clinical presentation, diagnosis, and prognosis of chronic laminitis. The Veterinary Clinics of North America, v.15, p. 375-394, 1999.

32. KUWANO, A. et al. Clinical and pathological study in a case of sinker laminitis developed after race and transportation. Journal of Equine Science, v. 8, p. 49-55, 1997.
33. NUMANS, S. R., van der WATERING, C. C. Navicular disease: podotrochlitis chronica and asseptica podotrochlosis. Equine Veterinary Journal, v. 5, p. 50, 1973.

34. ADAMS, O. R. Lameness in horses. Philadelphia: Lea \& Febiger, 1974. p. 250.

35. LOWE, J. E. Sex, breed and age incidence of navicular disease. In: ANNUAL CONVENTION OF THE AMERICAN ASSOCIATION OF EQUINE PRACTITIONERS, 20., 1976. Proceedings... p. 37.

36. ACKERMAN, N. Navicular disease in the horse: risk factors, radiographic changes and response to therapy. Journal of the American Veterinary Medical Association, v. 170, p. 183-187, 1977.

37. VALDEZ, H. et al. Navicular disease and the himlimbs of the horse. Journal of the American Veterinary Medical Association, v. 172, p. 291$292,1978$. 